\title{
Gingival Enlargement in Epileptic Patients on Phenytoin Therapy-An Evidence Based Approach
}

\begin{abstract}
Abhishek Singh Nayyar ${ }^{1 *}$, Mubeen Khan², GT Subhas ${ }^{3}$, B Nataraju ${ }^{4}$, Vijayalakshmi KR ${ }^{5}$ and Raghvendra BM ${ }^{6}$
${ }^{1}$ Department of Oral Medicine and Radiology, Government Dental College and Research Institute, Bangalore-560 002, Karnataka, India ${ }^{2}$ Department of Oral Medicine and Radiology, Government Dental College and Research Institute, Bangalore-560 002, Karnataka, India

${ }^{3}$ Department of Neurology, Government Dental College and Research Institute, Bangalore Medical College and Research Institute, Bangalore-560 002, Karnataka, India ${ }^{4}$ Department of Neurology, Bangalore Medical College and Research Institute, Bangalore-560 002, Karnataka, India

${ }^{5}$ Department of Oral Medicine and Radiology, Government Dental College and Research Institute, Bangalore-560 002, Karnataka, India

${ }^{6}$ Department of Internal Medicine, Bangalore Medical College and Research Institute, Bangalore-560 002, Karnataka, India

Abstract

Epilepsy is a common neurological disorder with recurrent seizures due to a chronic underlying process. Despite tremendous advances in the field of understanding the etio-pathogenesis of epilepsy, phenytoin still remains the drug of choice in its management. Chronic administration of phenytoin has been associated to have a number of adverse effects. Gingival enlargement is one such most often reported adverse drug sequela of long term phenytoin usage with exclusion of local factors contributing towards gingival enlargement. Hence, the study was planned to investigate the association of phenytoin and gingival enlargement seen in epileptic patients being treated with phenytoin. For this, 25 patients between the ages of 18-50 years clinically diagnosed with epilepsy prior to the start of phenytoin therapy were included based on selection criteria and written informed consents were obtained. Assessment of gingival status was done using the index originally described by Angelopoulos and Goaz and later modified by Miller and Damm [Go Index]. The results of the study confirmed a significant association between long term usage of phenytoin and the onset and severity of gingival enlargement in epileptic patients being treated with phenytoin. This is a baseline study; the results of the study suggest a higher incidence of gingival enlargement in phenytoin treated epileptic patients with local factors having a little role, if any, towards phenytoin induced gingival enlargement.
\end{abstract}

Keywords: Epilepsy; Gingival enlargement; Phenytoin

\section{Introduction}

Epilepsy is described as a chronic neurological disorder characterized by recurrent seizures of cerebral origin, presenting with episodes of sensory, motor or autonomic phenomenon with or without loss of consciousness [1]. A recent meta-analysis of published and unpublished studies puts an overall prevalence rate of epilepsy in India at 5.59 per 1,000 populations [2]. Despite the tremendous advances in the management of epilepsy, phenytoin still remains the drug of choice; however, the long term administration of phenytoin has been seen to lead to a number of adverse effects. Gingival enlargement is one such most frequently reported adverse effect of phenytoin [3]. Approximately $40-50 \%$ of the patients treated with phenytoin develop esthetically disfiguring enlargement of the gingivae. Whenever occurs, this adverse effect of phenytoin, lasts throughout the period of therapy and continues further with a severe reduction in the quality of life of the affected individual. The pseudopockets that are formed as a result of gingival enlargement increase plaque retentive areas which further predispose the patient towards an enhanced susceptibility for inflammatory changes in the gingivae, dental caries and periodontal diseases $[4,5]$. However, the etio-pathogenesis of phenytoin induced gingival enlargement is still not clearly understood, although, a number of studies indicate its multi-factorial etiology [6] including oral hygiene status of the affected epileptic patients. The present study was designed to investigate the association of phenytoin and gingival enlargement in epileptic patients being treated with phenytoin excluding the role of local factors, if any, with the maintenance of a meticulous oral hygiene.

\section{Materials and Methods}

\section{Source of data}

25 patients visiting the Department of Neurology, Victoria Hospital Bangalore during the period of Jan 2009 to Dec 2009 clinically diagnosed with epilepsy were selected prior to the start of phenytoin therapy based on the defined inclusion and exclusion criteria.

\section{Method of collection of data}

Selected epileptic patients, clinically diagnosed with epilepsy, were explained in detail about the planned study and written informed consents were obtained. These patients were subjected to a detailed history and a thorough clinical examination using a specially prepared proforma.

\section{Inclusion criteria}

1. Epileptic patients in the age group of 18-50 years,

2. Patients who were being started with phenytoin therapy,

3. Patients with full complement of teeth without any carious or periodontal involvement or any other pathological process in the teeth and the jaws.

\section{Exclusion criteria}

1. Epileptic patients with other systemic diseases,

*Corresponding author: Abhishek Singh Nayyar, Department of Oral Medicine and Radiology, Government Dental College and Research Institute, Bangalore- 560002 Karnataka, India, Tel: +91-9908688901; E-mail: singhabhishek.rims@gmail.com

Received March 09, 2012; Accepted April 17, 2012; Published April 20, 2012

Citation: Nayyar AS, Khan M, Subhas GT, Nataraju B, Vijayalakshmi KR, et al (2012) Gingival Enlargement in Epileptic Patients on Phenytoin Therapy-An Evidence Based Approach. J Neurol Neurophysiol 3:127. doi:10.4172/21559562.1000127

Copyright: (c) 2012 Nayyar AS, et al. This is an open-access article distributed under the terms of the Creative Commons Attribution License, which permits unrestricted use, distribution, and reproduction in any medium, provided the original author and source are credited. 
2. Epileptic patients with pre-existing gingival enlargements due to any reasons as idiopathic, inflammatory,neoplastic, endocrinal, chronic vitamin $\mathrm{C}$ deficiency, mouth breathing or pregnancy,

3. Epileptic patients on any type of pharmacologic therapy including multi-vitamins or, folate antagonists,

4. Epileptic patients who had history of dental treatment and trauma to teeth.

\section{Methodology}

Based on the selection criteria, 25 patients clinically diagnosed with epilepsy, were enrolled in the study with their written informed consents and then subjected to a thorough oral prophylaxis and routine hematological examination. After a gap of one week, these patients were thoroughly examined and their gingival status assessed using the index originally described by Angelopoulos and Goaz and later, modified by Miller and Damm [Go Index]. Before the start of study, the ethical clearance was obtained by the ethical committee of the institution as well as from Bangalore Medical College and Research Institute and Associated Hospitals.

Assessment of gingival enlargement: The gingival status was assessed using the [Go Index]. The height of gingival tissue was measured from the cemento-enamel junction to the free gingival margin. The grades for gingival enlargement were assessed in relation to the six anterior teeth in both the maxillary and mandibular arches based on the findings of the previous studies of the more common involvement of the anterior segments of the jaws, both on the mesial and the distal inter-proximal aspects and the greater score amongst them was selected to be included to refer to the peak effect of the drug. After a period of 2 months, the patients were reviewed and their gingival scores re-assessed (Figure 1) using the same criteria. The same procedure was repeated at the end of 6 months (Figure 2 and 3) of phenytoin therapy. Results were tabulated and subjected to statistically analysis.

\section{Method of statistical analysis}

The statistical analysis was done using paired t-test.

\section{Results}

The present study was designed in the Department of Oral Medicine and Radiology, Government Dental College and Research Institute, Bangalore during the period of Jan 2009 to Dec 2009 to investigate the association of phenytoin and phenytoin induced gingival enlargement in epileptic patients being treated with phenytoin. Selected epileptic patients to be enrolled in the study based on defined inclusion and exclusion criteria were explained in detail about the planned study and written informed consents were obtained.

The study consisted of a total of 25 patients with 19 male (76\%) and 6 female (24\%) patients (Figure 4). The mean age of the study group was 30.08 years with an age range of $18-50$ years. The mean age of the 19 male patients included in the study was 30.26 years with an age range of 18-50 years while for the 6 female patients with an age range of 20-36 years, the mean age was calculated to be 29.5 years (Figure 5).

The study revealed a higher incidence of gingival enlargement in phenytoin treated epileptic patients with the observation of gingival enlargement in all patients in the test group after 6 months of phenytoin administration, though to varying grades.

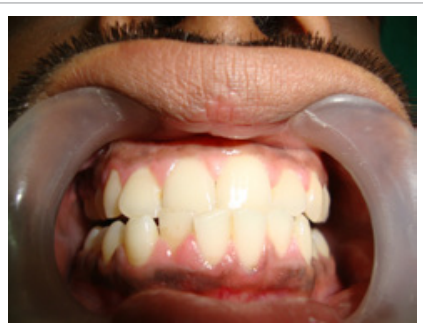

Figure 1: Clinical picture of gingival enlargement in a male epileptic patient (Grade 1) after 2 months of initiation of phenytoin therapy.

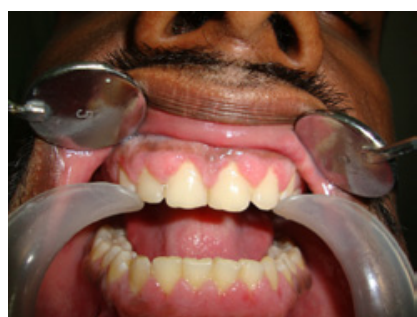

Figure 2: Clinical picture of gingival enlargement in a male epileptic patien (Grade 2) after 6 months of phenytoin therapy.

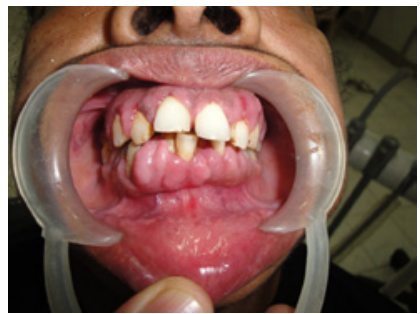

Figure 3: Clinical picture of bulbous gingival enlargement in a female epileptic patient with more prominent involvement of the interdental papillae (Grade 3) after 6 months of phenytoin therapy.

\section{Gender distribution in the study sample}

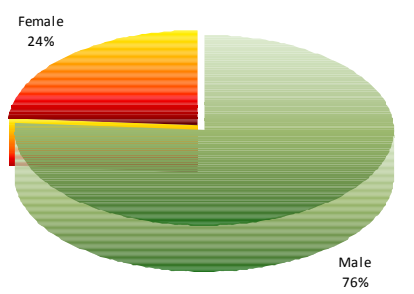

Figure 4: Pie diagram representing gender distribution in the study sample.

The gingival status was assessed using the index originally described by Angelopoulos and Goaz and later, modified by Miller and Damm [Go Index] prior to the start of and after 6 months of phenytoin therapy.

The average score of gingival enlargement prior to the start of phenytoin therapy in our study was found to be $1.7+/$ - 0.23 . In our study, an average grade of $1.74+/-0.21$ was obtained for the gingival enlargement in the 19 male patients included in the study with a range of 1.33-2.17 and $1.58+/-0.26$ with a range of $1.33-2.08$ for the 6 female patients (Table 1). Amongst these, 22 patients were found to have an approximate grade 2 while 3 patients with grade 1 gingival enlargement. 
Citation: Nayyar AS, Khan M, Subhas GT, Nataraju B, Vijayalakshmi KR, et al. (2012) Gingival Enlargement in Epileptic Patients on Phenytoin Therapy-An Evidence Based Approach. J Neurol Neurophysiol 3:127. doi:10.4172/2155-9562.1000127

Page 3 of 7

After 6 months of phenytoin therapy, the average grade for gingival enlargement was found to be $2.07+/-0.35$. After 6 months of phenytoin treatment, average grade for gingival enlargement was found to be $2.04+/-0.38$ with a range of $1.17-2.67$ in the male patients while $2.14+/-0.23$ with a range of $1.83-2.42$ for the female patients (Table 2). Amongst these, 20 patients were found to have nearing grade 2, 2 patients, grade 1 and 3 patients, grade 3 gingival enlargement.

The statistical analysis was done using paired t-test. The results arrived found the increase in mean gingival enlargement from before to after 6 months of phenytoin therapy to be statistically significant with a p-value of less than 0.001 with the level of significance kept at 0.05 (Table 3).

Discussion - Despite tremendous advances in the management of epilepsy in the recent decade, the anti-epileptic drug phenytoin still remains the prime drug of choice in the management of epileptic patients in India $[7,8]$.

Chronic administration of phenytoin has been associated with a number of adverse effects $[9,10]$. Gingival enlargement is one such most often reported adverse drug consequence of long term phenytoin usage [11]. Drug-induced gingival enlargement (DIGE) associated with chronic use of the anti-epileptic drug phenytoin was first reported in 1939 by Kimball [12]. In the same year, Faurbye [13] and in 1959, Strean and Leoni [14] suggested that the alkalinity of phenytoin might be the cause of the gingival side effect. In 1948, Brandon [15] hypothesized that phenytoin had a direct action on the gingival tissues. In 1975, Angelopoulos [6] argued that phenytoin induced degranulation of mast cells which resulted in the generation of a substance that increased collagen formation. Larmas [16] in 1976, suggested that phenytoin had a proliferating effect primarily on the basal cell layer of the oral epithelium thus increasing the epithelium-connective tissue interface area, which was confirmed by Hassel et al. [17]. Furthermore, the oral

Mean age of the study sample and according to gender

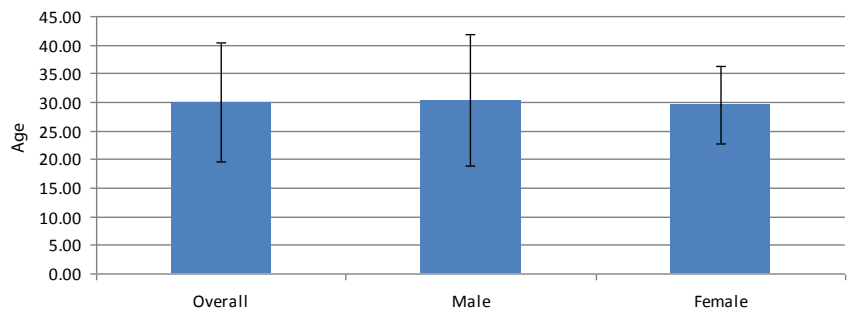

Figure 5: Bar diagram representing mean age in the study sample.

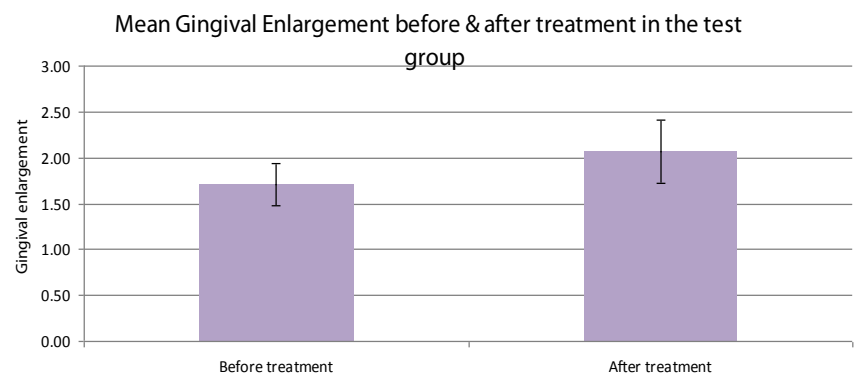

Figure 6: Bar diagram representing mean grades for gingival enlargement in the test group before and after 6 months of phenytoin treatment.

\begin{tabular}{|l|c|l|l|l|l|l|}
\hline $\begin{array}{l}\text { Gingival } \\
\text { Enlargement-Before } \\
\text { treatment }\end{array}$ & Mean & Std dev & Median & Min & Max & Range \\
\hline Overall & 1.70 & 0.23 & 1.7 & 1.33 & 2.17 & 0.84 \\
\hline Male & 1.74 & 0.21 & 1.8 & 1.33 & 2.17 & 0.84 \\
\hline Female & 1.58 & 0.26 & 1.5 & 1.33 & 2.08 & 0.75 \\
\hline
\end{tabular}

Table 1: Table depicting overall mean grades for gingival enlargement with range, and same in case of male and female patients before phenytoin treatment.

\begin{tabular}{|l|l|l|l|l|l|l|}
\hline $\begin{array}{l}\text { Gingival Enlargement- } \\
\text { After treatment }\end{array}$ & Mean & Std dev & Median & Min & Max & Range \\
\hline Overall & 2.07 & 0.35 & 2.1 & 1.17 & 2.67 & 1.50 \\
\hline Male & 2.04 & 0.38 & 2.0 & 1.17 & 2.67 & 1.50 \\
\hline Female & 2.14 & 0.23 & 2.2 & 1.83 & 2.42 & 0.59 \\
\hline
\end{tabular}

Table 2: Table depicting overall mean grades for gingival enlargement with range, and same in case of male and female patients after 6 months of phenytoin treatment.

\begin{tabular}{|l|l|l|l|l|l|l|}
\hline Group & $\mathrm{n}$ & Mean & Std dev & $\begin{array}{l}\text { Mean } \\
\text { difference }\end{array}$ & $\mathrm{t}$ & P-Value \\
\hline Before treatment & 25 & 1.70 & 0.23 & -0.365 & -4.807 & $<0.001^{*}$ \\
\hline After treatment & 25 & 2.07 & 0.35 & & & \\
\hline
\end{tabular}

*denotes significant difference

Table 3: Table depicting comparison of mean grades of gingival enlargement in the test group before and after 6 months of phenytoin treatment.

epithelium may have an inducing effect on the underlying fibroblasts, in which specifically alkaline phosphatase may be involved.

In 1977, Angelopoulos [6] speculated that phenytoin induced gingival enlargement was due to an end-organ folic acid deficiency, which could lead the gingival tissues susceptible to inflammation by causing degenerative changes in the gingival sulcular epithelium, the main physical barrier against local irritants.

Apart from elevated production of connective tissue matrix in the phenytoin induced gingival enlargements, in-vitro research has also focused on the possibility of an accumulation of redundant tissue as a result of inhibition of matrix breakdown. While cells from phenytoin induced gingival enlargements have been seen to synthesize and secrete elevated quantities of collagenase, the majority of the enzyme produced by such cells has been found to be inactive. Also, Hassell et al. [17] had characterized the collagen types present in normal gingival tissues and in phenytoin induced gingival enlargements and detected that the latter manifested less of type I and more of type III collagen. Furthermore, it has been demonstrated in vitro that phenytoin directly inhibits the conversion of inactive collagenase pro-enzyme to the active form.

Ultra structurally, the fibroblasts of phenytoin induced gingival enlargements exhibit a decrease in the volume density of rough endoplasmic reticulum and a smaller nuclear to cytoplasmic ratio with possible implication of this being that while the fibroblasts of the phenytoin treated gingival enlargements are synthetically active, they are possibly less active in degradative functions thus accounting for the alterations in the gingival connective tissue matrix [9].

In our study, 25 patients between the ages of 18-50 years visiting the Department of Neurology, Victoria Hospital, Bangalore during the period of Jan 2009 to Dec 2009 clinically diagnosed with epilepsy were 
selected prior to the start of phenytoin therapy based on the defined inclusion and exclusion criteria. The study consisted of a total of 19 male $(76 \%)$ and 6 female $(24 \%)$ patients.

The mean age of the study group was 30.08 years with an age range of 18-50 years. The mean age of the 19 male patients included in the study was 30.26 years with an age range of 18-50 years while for the 6 female patients with an age range of 20-36 years, the mean age was calculated to be 29.5 years.

Numerous reports suggest that phenytoin induced gingival enlargement is more commonly seen in younger age groups. This is in concordance with the observations of the several epidemiological studies. Also, both genders have been reported to be equally susceptible to phenytoin induced gingival enlargement in the literature [11]. The above mentioned observations were confirmed in our study as well since most of the patients with more severe gingival enlargements were in the age group of 20-40 years and the cases were reported with equal frequency in both the male and female patients.

The study revealed a high incidence of gingival enlargement in epileptic patients on phenytoin therapy with the observation of varying grades of gingival enlargement in all patients in test group after 6 months of phenytoin administration. The incidence of phenytoin induced gingival enlargement as reported by a study conducted by Kimball was found to be $57 \%$ while other studies conducted in relation to incidence of phenytoin induced gingival enlargement have revealed wide incidence ranges of $20-40 \%[18,19]$ in some studies to $6-79 \%$ in others [19-24] while 3-93\% in few other studies [25,26] and 50\% in institutionalized epileptic patients as reported in the literature. The incidence of gingival overgrowth in the normal population has been reported to be between $4-7.5 \%$ [27]. This wide range of variability may be attributed to the small number of the cases reported in some publications to large variations in phenytoin dosages to variations in the length of phenytoin exposure and to differences in the age of the patients included in the various studies as well.

Drug induced gingival enlargement normally begins at the interdental papillae and is more frequently found in the anterior segments of the jaws though it often involves all the surfaces of teeth and is generalized in its distribution $[9,23,24]$. Gradually, gingival lobulations are formed that may appear inflamed or more fibrotic in nature depending on the degree of local factors' induced secondary inflammatory changes.

Currently, more than 15 drugs have been identified as possible causative agents, including oral contraceptives $[8,28]$. However, there are 3 classes of drugs that are well-established causes of gingival enlargement, being responsible for most cases: anti-epileptic agents, anti-hypertensive calcium antagonists and immunosuppressant cyclosporine. One property that is common to these 3 classes of drugs is that they all directly affect cellular calcium metabolism. Since cellular production of collagenase is modulated by calcium influx, fibroblasts from patients treated with these drugs may produce an inactive form of collagenase, being responsible for an increase in extra-cellular matrix [18].

The precise mechanism by which drug-induced gingival enlargement occurs is still not completely understood, although a number of hypotheses have been suggested [6]. Phenytoin probably interacts with a subtype of susceptible fibroblasts, cyclosporine affects the metabolism of these cells and nifedipine enhances this effect reducing their metabolism $[8,29]$. Several factors may influence the relationship between the various implicated drugs and components of the gingival tissues, including: age, genetic predisposition, pharmacokinetic variables, drug-induced alterations in gingival connective tissue homeostasis, ultra structural factors and inflammatory changes, drug-induced action on growth factors, etc. Three significant factors which are considered to be the most important etiological factors in the expression of these gingival changes include: drug variables, plaque-induced inflammatory changes in the gingival tissues and genetic factors - the latter determining the heterogeneity of the gingival fibroblasts [30].

Also, some drugs induce a direct effect on a subgroup of fibroblasts, named "responders", that are apparently genetically determined to be sensitive to the drug causing gingival growth. Such drugs produce a decrease in calcium influx (due to alterations in calcium-sodium exchange), which causes a decrease in cellular folic acid uptake (producing a localized folate deficiency) thus, limiting the production of the collagenase-activating enzyme (the active form of collagenase) [31]. Also, since the presence of inflammation secondary to dental plaque causes proliferative increases in connective tissue, the catabolic ability of collagenase is saturated, and the inhibited degradation of the extra cellular matrix causes a local accumulation of this matrix $[4,11]$. However, several other factors may be involved in drug induced gingival enlargement including the so-named androgenic hormones [32].

Brown et al. (1991) [33] have pointed out in this entity several factors: increase of sulphatid glicosamines, immunoglobulins, epithelial growth factor; calcium and sodium rupture efflux in fibroblasts, folic acid and collagenase deficiency [4,31]. Saito et al. [34] have shown by immunohistochemistry studies in gingival enlargement caused by phenytoin and nifedipine that the increase in beta-growing factor, basic growing fibroblast factor, its receptors and glicosaminoglicans heparan sulphate are involved; this was confirmed in other studies [31,33,35].

The clinical presentation of gingival hypertrophic and inflamed tissues is associated with specific macrophagic phenotypic picture that express beta- citocine IL-1 in tissues or platelet derived growth factor. Iacopino et al. [36] and Saito et al. [37] have speculated that p53 protein expression in drug induced gingival enlargement suggests that its pathogenesis is involved with DNA abnormalities [31,36,37]. Reduction of IgA salivary levels has also been accounted for the cause of gingival enlargement induced by phenytoin but it was not confirmed, as well as alteration of sub-gingival microflora [31,38-42].

Dose-dependent correlations with the severity of gingival overgrowth are weak, but decreased drug use in general results in reduced severity of gingival pathology. For example, phenytoin was reported to effuse into crevicular fluid without any correlation to the incidence of overgrowth, while no direct link was shown between overgrowth and the concentrations of phenytoin and metabolites. A more recent study supports a correlation between diminished metabolism of phenytoin in affected individuals and overgrowth, but this has not been confirmed [31].

Age, gender, concomitant medication with multiple drugs, local factors such as plaque accumulation, and genetic disposition are additional complicating risk factors in drug-induced gingival overgrowth [31].

All the clinical features of phenytoin induced gingival enlargement were confirmed in our study wherein we observed a predominantly firm and fibrotic nature of the gingival enlargement in most of the patients 
with local factors' induced secondary inflammatory changes having a minor role, if any, to play in the clinical picture of the phenytoin induced lesions of gingival enlargement as the oral hygiene was meticulously maintained. The observations of our study also revealed that the interdental papillae were the most common sites of involvement for the phenytoin induced gingival enlargements. The tissues affected were though not subjected to a detailed histo-pathological analysis as the patients were not subjected to surgical therapeutic options for the treatment that carries a high probability for recurrence $[8,9,11]$.

Also, significant was the observation that the gingival enlargement induced by phenytoin was usually generalized with involvement of all surfaces of the teeth in all the quadrants but was more severe in the anterior segments of the jaws as per the observations of the prior studies possibly because of a relative lack of oral hygiene maintenance in these areas of the jaws.

A review of the gingival enlargement indices proposed in the literature clearly demonstrates their diversity, from the most simple gingival enlargement index proposed to the most elaborate one. Different authors have used different criteria for grading the gingival enlargement in their studies however there is no universal criteria that can be adopted for the same as every criteria has a more or less subjective methodological approach for the assessment of gingival enlargement and depends on the author's discretion for following the same. The majority of the indices used to quantify gingival enlargement are difficult to reproduce because they lack an objective criteria to differentiate between the degree of horizontal and vertical overgrowth.

In our study, the gingival status was assessed using the index originally described by Angelopoulos and Goaz and later, modified by Miller and Damm [Go Index]. The height of gingival tissue was measured from the cemento-enamel junction to the free gingival margin. The grades for gingival enlargement were assessed in relation to the six anterior teeth in both the maxillary and mandibular arches. The enlargement was recorded both on the mesial and the distal interproximal aspects and the greater score amongst them was selected to be included to refer to the peak effect of the drug. Other criteria for assessing the gingival enlargement were not followed for their being with too extensive methodologies and yet with highly subjective nature of assessment of gingival enlargements.

In our study, an average grade of $1.74+/-0.21$ was obtained for the gingival enlargement in the 19 male patients included. The average grade for the 6 female patients was found to be1.58+/- 0.26 prior to the start of phenytoin therapy. Amongst these, 22 patients were found to have an approximate grade 2 with 3 patients having grade 1 gingival enlargement.

After 6 months of phenytoin treatment, average grade for gingival enlargement was found to be $2.04+/-0.38$ with a range of 1.17-2.67 in the male patients while $2.14+/-0.23$ with a range of $1.83-2.42$ for the female patients. Amongst these, 20 patients were found to have nearing grade 2 while 3 patients were found to have grade 3 gingival enlargement.

The results obtained could not be compared with the observations of other studies as the indices followed were either different, modified Harris and Ewalt index in a study conducted by Prasad et al. [43] on the role of folic acid in the prevention of phenytoin induced gingival enlargement on sixty epileptic children in the age range of 8-13 years [43] or even using the same index in a cross sectional study conducted by Brunet et al. on 59 patients on anti-epileptic medications using the
GO and MB indices [11] owing to the subjectivity of the assessment procedure and a lack of reproducibility with local factors further playing a confounding role in the observations.

Treatment of the gingival overgrowth lesion itself can be complicated due to the superimposed inflammation on the fibrotic tissue enlargement. Traditionally, periodontal therapy offers removal of the inflammatory component of the overgrowth through scaling and gingival curettage, followed by excision of the overgrown gingiva $[12,17,44]$. For patients with severe gingival overgrowth and who require continuous drug therapy for medical reasons, gingivectomy must be repeated periodically due to the recurrent nature of druginduced gingival overgrowth.

Nevertheless, there is a controversy whether therapy with folic acid would have a therapeutic effect on phenytoin-induced gingival overgrowth. Inoue and Harrison suggested that patients taking phenytoin and a supplement of folic acid had gingival overgrowth prevented or eliminated [45]. Some other studies showed that patients receiving folate had lower recurrence of gingival enlargement after its surgical removal $[46,33]$. In a recent study, Prasad et al. evaluated 60 patients and also concluded that therapy with folic acid is able to delay and minimize phenytoin-induced gingival overgrowth [43]. In opposition to these data, Brown et al. [4] reported that folic acid was not efficient as a sole therapeutic agent in the reduction of phenytoininduced gingival overgrowth [47]. Similarly, Majola et al. [47] found that serum folate levels did not have any significant association with phenytoin-induced gingival overgrowth [48]. These contradictory findings are not sufficient to indicate folic acid therapy as a reliable alternative in the treatment of phenytoin-induced gingival overgrowth.

In drug-induced gingival enlargements, reversing and preventing gingival enlargement is as easy as ceasing drug therapy. However, this is not always feasible; in such a situation, alternative drug therapy may be employed, if possible, to avoid this deleterious side effect. In the case of immuno-suppression, tacrolimus is an available alternative which results in much less severe gingival overgrowth than cyclosporin, but is similarly as nephrotoxic [49]. The dihydropyridine derivative isradipidine can replace nifedipine for some uses of calcium channel blocking and does not induce gingival overgrowth [50].

However, this is a preliminary study; the results of the study suggest a higher incidence and severity of gingival enlargement in phenytoin treated epileptic patients (Graph 3). No available published reports with similar methodology have been found in the literature. Hence, this study gives a scope for further studies with larger sample size to conclude the results.

\section{Conclusion}

This is a preliminary study which aimed to investigate the association of phenytoin and phenytoin induced gingival enlargement in epileptic patients being treated with phenytoin. The results of the study suggests a higher incidence and severity of gingival enlargement in phenytoin treated epileptic patients with local factors having a little role, if any, towards phenytoin induced gingival enlargement . However, since this is only a baseline study and the tissues affected were not subjected to a detailed histo-pathological analysis as the patients were not subjected to surgical therapeutic options for the treatment that carries a high probability for recurrence, the results of the study encourage for further studies with larger sample size for the confirmation of the nature of histology of phenytoin induced gingival enlargement in phenytoin treated epileptic patients as well 
Citation: Nayyar AS, Khan M, Subhas GT, Nataraju B, Vijayalakshmi KR, et al. (2012) Gingival Enlargement in Epileptic Patients on Phenytoin Therapy-An Evidence Based Approach. J Neurol Neurophysiol 3:127. doi:10.4172/2155-9562.1000127

as the estimation of serum and salivary levels of phenytoin and folic acid and tissue levels of folates to substantiate the exact role of systemic and tissue levels of phenytoin and folic acid in the etio-pathogenesis of phenytoin-induced gingival overgrowth.

Contributions from the authors: Literature search, manuscript preparation, manuscript editing and manuscript review.

\section{Ethical Declaration}

The study has been approved by the ethical committee appointed by the Government Dental College and Research Institute, Bangalore and Bangalore Medical College and Research Institute, Bangalore and has therefore been performed in accordance with the ethical standards laid down in the 1975 declaration of Helsinki and its later amendments in 2000 after a written informed consent from the patients for their inclusion in the study. Details that might disclose the identity of the patient have been omitted.

\section{Acknowledgement}

We thank all the people who directly and indirectly contributed for the study as the study required intense efforts from the people outside our Department including Department of Neurology and Department of Clinical Biochemistry, Bangalore Medical College and Research Institute and Associated Hospitals.

\section{References}

1. Sridharan R (2002) Epidemiology of epilepsy. Current Science 82: 664-670.

2. Sridharan R, Murthy BN (1999) Prevalence and pattern of epilepsy in India Epilepsia 40: 631-636.

3. Hassell TM (1981) Epilepsy and the Oral Manifestations of Phenytoin Therapy. Monographs in Oral Science 9: 1-205.

4. Brown RS, Sein P, Corio R, Bottomley WK (1990) Nitrendipine- induced gingival hyperplasia: first case report. Oral Surg Oral Med Oral Pathol 70: 593-596.

5. Whitehead N, Reyner F, Lindernbaum J (1973) Megaloblastic changes in the cervical epithelium. Association with oral contraceptive therapy and reversal with folic acid. JAMA 226: 1421-1424.

6. Angelopoulos AP (1975) Diphenylhydantoin gingival hyperplasia: A clinicopathological review of Incidence, clinical features and histopathology. Dent J 41: 103-106

7. Scheinfeld N (2003) Phenytoin in cutaneous medicine: its uses, mechanisms and side effects. Dermatol Online J 9: 6 .

8. Hassessian A, Guimarães Júnior J, Marcucci G (2003) Freqüência da hyperplasia gengival medicamentosa em 48 pacientes tratados pela nifedipina/ Gingival hyperplasia frequency in 48 patients under nifedipine treatment. Rev. ABO nac 11: 28-32.

9. Hassell TM, Burtner AP, McNeal D, Smith RG (2000) Hypertrophic Oral problems and genetic aspects of individuals with epilepsy. Periodontol 2000 6: 68-78.

10. Ciancio SG, Yaffe SJ, Catz CC (1972) Gingival hyperplasia and diphenylhydantoin. J Periodontol 43: 411-414.

11. Brunet L, Miranda J, Farré M, Berini L, Mendieta C (1996) Gingival enlargement induced by drugs. Drug Saf 15: 219-231.

12. Kimball OP (1939) The treatment of epilepsy with sodium diphenylhydantoinate. J Am Med Assoc 112: 1244-1245

13. Faurbye A (1939) Rehandling of epilepsy med diphenylhydantoin. Ugeskr Laeg 101: $1350-1354$

14. Strean LR, Leoni E (1959) Dilantin gingival hyperplasia. Newer concepts related to etiology and treatment. NY St Dent J 25: 339-347.

15. Brandon SA (1948) Treatment of hypertrophy of the gingival tissue caused by Dilantin sodium therapy. J Am Dent Assoc 37: 732-735.

16. Larmas L (1977) A comparative enzyme histochemical study of hydantoin induced hyperplastic and normal human gingiva. Proc Finn Dent Soc 73: 1-27.

17. Hassell TM, Page RC, Narayanan AS, Cooper CG (1976) Diphenylhydantoin (Dilantin) gingival hyperplasia: Drug-induced abnormality of connective tissue. Proc Natl Acad Sci U S A 73: 2909-2912.

18. Cals MJ, Bories PN, Devanlay M, Desveaux N, Luciani L, et al. (1994)
Extensive laboratory assessment of nutritional status in fit, health-conscious, elderly people living in the Paris area. Research Group on Aging. J Am Col Nutr 13: 646-657.

19. Weggemans RM, de Groot LC, Haller J (1997) Factors related to plasma folate and vitamin B12.The SENECA study. Int J Food Sci Nutr 48: 141-150.

20. Benton D, Haller J, Fordy J (1997) The vitamin status of young British adults Int J Vitam Nutr Res 67: 34-40

21. Rosenberg IH, Bowman BB, Cooper BA, Halsted CH, Lindenbaum J (1982) Folate nutrition in the elderly. Am J Clin Nutr 36: 1060-1066.

22. Ferro-Luzzi A, Mobarhan S, Maiani G, Scaccini C, Sette S, et al. (1988) Habitual alcohol consumption and nutritional status of the elderly. Eur J Clin N utr 42: 5-13.

23. Marshall RI, Bartold PM (1999) A clinical review of drug induced gingival overgrowths. Aust Dent J 44: 219-232.

24. Hallmon WW, Rossmann JA (1999) The role of drugs in the pathogenesis of gingival overgrowth: A collective review of current concepts. Periodontol 2000 21: 176-196.

25. Lennox WG (1940) The drug therapy of epilepsy. JAMA 114: 1347-1354.

26. Kapur RN, Girgis S, Little TM, Masotti RE (1973) Diphenylhydantoin-induced gingival hyperplasia: its relationship to dose and serum level. Dev Med Child Neurol 15: 483-487.

27. Wiebe S, Blume WT, Girvin JP, Eliasziw M (2001) A randomized, controlled trial of surgery for temporal-lobe epilepsy. N Engl J Med 345: 311-318.

28. Silverstein LH, Garnick JJ, Szikman M, Singh B (1997) Medication induced gingival enlargement: a clinical review. Gen Dent 45: 371-376.

29. Guimarães Jr J (2007) Hiperplasia gengival medicamentosa - Parte I. Journal of Epilepsy and Clinical Neurophysiology 13: 33-36.

30. Seymour RA, Thomason JM, Ellis JS (1996) The pathogenesis of drug induced gingival overgrowth. J Clin Periodontol 23: 165-175.

31. Correa JD, Queiroz-Junior CM, Costa JE, Teixeira AL, Silva TA (2011) Phenytoin-induced gingival overgrowth: a review of the molecular, immune, and inflammatory features. ISRN Dent 2011: 497850.

32. Sooriyamoorthy M, Gower DB, Eley BM (1990) Androgen metabolism in gingival hyperplasia induced by nifedipine and cyclosporin. J Periodontal Res 25: $25-30$

33. Brown RS, Di Stanislao PT, Beaver WT, Bottomley WK (1991) The administration of folic acid to institutionalized epileptic adults with phenytoininduced gingival hyperplasia. A double-blind, randomized, placebo-controlled, parallel study. Oral Surg Oral Med Oral Pathol 71: 565-568.

34. Saito K, Mori S, Iwakura M, Sakamoto S (1996) Immunohistochemica localization of transforming growth factor beta, basic fibroblast growth facto and heparan sulphate glycosaminoglycan in gingival hyperplasia induced by nifedipine and phenytoin. J Periodontal Res 31: 545-555

35. Kuru L, Yilmaz S, Kuru B, Kose KN, Noyan U (2004) Expression of growth factors in the gingival crevice fluid of patients with phenytoin induced gingival enlargement. Arch Oral Biol 49: 945-950.

36. lacopino AM, Doxey D, Cutler CW, Nares S, Stoever K, et al. (1997) Phenytoin and cyclosporine $A$ specifically regulate macrophage phenotype and expression of platelet-derived growth factor and interleukin-1 in vitro and in vivo: possible molecular mechanism of drug-induced gingival hyperplasia. J Periodontol 68 : 73-83.

37. Saito K, Mori S, Tanda N, Sakamoto S (1999) Expression of p53 protein and $\mathrm{Ki}-67$ antigen in gingival hyperplasia induced by nifedipine and phenytoin. $J$ Periodontol 70: 581-586.

38. Nikfarjam J, Pourpak Z, Shahrabi M, Nikfarjam L, Kouhkan A, et al. (2004) Ora manifestations in selective IgA deficiency. Int J Dent Hyg 2: 19-25.

39. Aarli JA (1976) Phenytoin induced depression of salivary IgA and gingival hyperplasia. Epilepsia 17: 283-291.

40. Smith QT, Hamilton MJ, Biros MH, Pihlstrom BL (1979) Salivary and plasma IgA of seizure subjects receiving phenytoin. Epilepsia 20: 17-23.

41. Akalin FA, Yavuzyilmaz E, Ersoy F, Kalfa Z, Muftuoglu M (1993) Immunoglobulin A levels in serum and saliva of patients treated with phenytoin. J Nihon Univ Sch Dent 35: 10-15. 
Citation: Nayyar AS, Khan M, Subhas GT, Nataraju B, Vijayalakshmi KR, et al. (2012) Gingival Enlargement in Epileptic Patients on Phenytoin Therapy-An Evidence Based Approach. J Neurol Neurophysiol 3:127. doi:10.4172/2155-9562.1000127

42. Takada K, Sugiyama H, Umezawa K, Mega J, Hirasawa M (2003) The subgingival microflora in phenytoin induced gingival hyperplasia. J Periodontal Res 38: 477-481

43. Prasad VN, Chawla HS, Goyal A, Gauba K, Singhi P (2004) Folic acid and phenytoin induced gingival overgrowth-ls there a preventive effect. J Indian Soc Pedod Prev Dent 22: 82-91.

44. Inoue F, Harrison JV (1981) Folic acid and phenytoin hyperplasia. Lancet 2: 86.

45. Williams C, Poppell T, Brock D, Low S. Phenytoin induced gingival overgrowth: effect of folic acid supplementation. Clinical Research 35: 61.

46. Poppell TD, Keeling SD, Collins JF, Hassell TM (1991) Effect of folic acid on recurrence of phenytoin-induced gingival overgrowth following gingivectomy. $J$ Clin Periodontol 18: 134-139.
47. Majola MP, McFadyen ML, Connolly C, Nair YP, Govender M, et al. (2000) Factors influencing phenytoin induced gingival enlargement. J Clin Periodonto 27: 506-512.

48. Brandon SA (1948) Treatment of hypertrophy of the gingival tissue caused by Dilantin sodium therapy. J Am Dent Assoc 37: 732-735.

49. Spencer CM, Goa KL, Gillis JC (1997) Tacrolimus. An update of its pharmacology and drug efficacy in the management of organ transplantation. Drugs 54: 925-975.

50. Westbrook P, Bednarczyk EM, Carlson M, Sheehan H, Bissada NF (1997) Regression of nifedipine-induced gingival hyperplasia following switch to a same class calcium channel blocker, isradipine. J Periodontol 68: 645-650. 